\title{
COINCIDENCE AND COMMON FIXED POINTS OF WEAKLY RECIPROCALLY CONTINUOUS AND COMPATIBLE HYBRID MAPPINGS VIA AN IMPLICIT RELATION AND AN APPLICATION
}

\author{
S. CHAUHAN, M. IMDAD, Z. KADELBURG AND C. VETRO
}

\begin{abstract}
Using the hybrid version of the notion of weakly reciprocally continuous pairs of mappings due to Gairola et al. [Coincidence and fixed point for weakly reciprocally continuous single-valued and multivalued maps, Demonstratio Math. (2013/2014), accepted], we prove a coincidence and common fixed point theorem for a hybrid pair of compatible mappings via an implicit relation. Our main result improves and generalizes a host of previously known theorems. As an application, we give a homotopy theorem which supports our main result.
\end{abstract}

\section{INTRODUCTION AND PRELIMINARIES}

The celebrated Banach Contraction Principle is one of the most fruitful results in nonlinear functional analysis. In 1969, Nadler [11] proved a multivalued version of the Banach Contraction Principle which is also known as the Nadler Multi-valued Contraction Principle. It has various applications within and beyond mathematics (see, for instance, $[2,22]$ ). It provides techniques for solving functional inclusions and optimization problems (see [4]). Hybrid fixed point theory for nonlinear single-valued and multi-valued mappings is a development within the multivalued fixed point theory which has already made considerable progress. In recent years, there has been vigorous and intense research activity in multi-mapping fixed point theory (including hybrid fixed point results) and by now there exists an extensive literature on this specific theme. Here, it can be specifically pointed out that hybrid fixed theorems have numerous applications in science and engineering.

2010 Mathematics Subject Classification. 47H09, 47H10, 54H25.

Key words and phrases. Hybrid pair of mappings, compatible mappings, non-compatible mappings, weak reciprocal continuity, coincidence point, common fixed point, implicit relation.

Copyright (c) 2015 by ANUBiH. 
In the following lines, we present some definitions and their implications which will be utilized throughout this paper.

Let $(X, d)$ be a metric space. Then, in the line with Nadler [11], we adopt the following:

(1) $C L(X)=\{A: A$ is a non-empty closed subset of $X\}$,

(2) $C B(X)=\{A: A$ is a non-empty closed and bounded subset of $X\}$,

(3) For non-empty closed and bounded subsets $A, B$ of $X$ and $x \in X$,

$$
\begin{gathered}
d(x, A)=\inf \{d(x, a): a \in A\} \text { and } \\
H(A, B)=\max \{\sup \{d(a, B): a \in A\}, \sup \{d(b, A): b \in B\}\} .
\end{gathered}
$$

It is well known that $C B(X)$ is a metric space with the distance $H$ which is known as the Hausdorff-Pompeiu metric on $C B(X)$. The following terminology is also standard.

Let $(X, d)$ be a metric space with $f: X \rightarrow X$ and $T: X \rightarrow C B(X)$. Then

(1) a point $x \in X$ is a fixed point of $f($ resp. $T)$ if $x=f x($ resp. $x \in T x)$. The set of all fixed points of $f$ (resp. $T$ ) is denoted by $F(f)$ (resp. $F(T)$,

(2) a point $x \in X$ is a coincidence point of $f$ and $T$ if $f x \in T x$. The set of all coincidence points of $f$ and $T$ is denoted by $C(f, T)$,

(3) a point $x \in X$ is a common fixed point of $f$ and $T$ if $x=f x \in T x$. The set of all common fixed points of $f$ and $T$ is denoted by $F(f, T)$,

(4) for $A \subset X, T(A)$ stands for the set $\bigcup_{x \in A} T x$. In particular, $T(X)=$ $\bigcup_{x \in X} T x$.

The notions of commutativity and weak commutativity for a hybrid pair of mappings on metric spaces were defined by Kaneko $[9,10]$. Thereafter, Singh et al. [19] weakened the notion of weak commutativity by introducing the notion of compatible mappings for a hybrid pair of mappings and obtained some common fixed point theorems for nonlinear hybrid contractions. This way of proving new results continue to attract the attention of many researchers of this domain, where it can be observed that under compatibility the fixed point results often require continuity of one of the underlying mappings. In 2002, Singh and Mishra [20] extended the notion of reciprocal continuity (due to Pant [12]) to a hybrid pair of mappings and established some common fixed point theorems in metric spaces. Most recently, Gairola et al. [5] defined a more general notion, namely weak reciprocal continuity (due to Pant et al. [13]) for a hybrid pair of mappings and proved common fixed point theorems for non-expansive mappings. The technical definitions of the earlier mentioned notions are described in the following lines.

Definition 1. Let $(X, d)$ be a metric space with $f: X \rightarrow X$ and $T: X \rightarrow$ $C L(X)$. A hybrid pair of mappings $(f, T)$ is said to be: 
(1) commuting on $X[9]$ if $f T x \subseteq T f x$ for all $x \in X$,

(2) compatible [19] if $f T x \in C L(X)$ for all $x \in X$ and $\lim _{n \rightarrow \infty} H\left(T f x_{n}\right.$, $\left.f T x_{n}\right)=0$, whenever $\left\{x_{n}\right\}$ is a sequence in $X$ such that $T x_{n} \rightarrow A \in$ $C L(X)$ and $f x_{n} \rightarrow t \in A$, as $n \rightarrow \infty$,

(3) reciprocally continuous on $X$ (resp., at $t \in X$ ) [20] if $f T x \in C L(X)$ for each $x \in X$ (with $f T t \in C L(X)) \lim _{n \rightarrow \infty} f T x_{n}=f A$ and $\lim _{n \rightarrow \infty} T f x_{n}=T t$ whenever $\left\{x_{n}\right\}$ is a sequence in $X$ such that $\lim _{n \rightarrow \infty} T x_{n}=A \in C L(X), \lim _{n \rightarrow \infty} f x_{n}=t \in A$,

(4) weakly reciprocally continuous on $X$ (resp., at $t \in X$ ) [5] if $f T x \in$ $C L(X)$ for each $x \in X$ (with $f T t \in C L(X))$ and $\lim _{n \rightarrow \infty} f T x_{n}=$ $f A$ or $\lim _{n \rightarrow \infty} T f x_{n}=T t$ whenever $\left\{x_{n}\right\}$ is a sequence in $X$ such that $\lim _{n \rightarrow \infty} T x_{n}=A \in C L(X), \lim _{n \rightarrow \infty} f x_{n}=t \in A$,

(5) coincidentally idempotent [7] if $f f v=f v$ for every $v \in C(f, T)$, i.e., $f$ is idempotent at the coincidence points of $f$ and $T$,

(6) occasionally coincidentally idempotent [14] if $f f v=f v$ for some $v \in C(f, T)$.

For more details on systematic comparison and illustrations of earlier described notions, we refer to $[5,7-10,19,20]$.

Lemma 1 ( $[11])$. Let $P, Q \in C L(X)$ and $k>1$. Then for each $p \in P$, there exists a point $q \in Q$ such that $d(p, q) \leq k H(P, Q)$.

In this paper, an attempt has been made to derive common fixed point theorems for a hybrid pair of mappings using weak reciprocal continuity with occasionally coincidentally idempotent property besides employing an implicit relation and compatibility. The presented theorems extend and unify various known fixed point results. In addition, we give a homotopy theorem using our results.

\section{IMPLICIT RELATIONS}

In the recent past, implicit functions has been utilized to prove unified common fixed points results covering various kinds of contraction conditions in one go (see, for example, $[3,21]$ and references therein). In fact, this idea was initiated by Popa $[15,16]$ where the author introduced an implicit function which covers a variety of contraction classes. In [18], Popa and Patriciu introduced the following implicit function and utilize the same to prove some coincidence and common fixed point results for hybrid pairs of mappings covering several contraction conditions in one go.

In what follows, $\Phi$ denotes the set of all continuous functions $\phi: \mathbb{R}_{+}^{6} \rightarrow \mathbb{R}$ satisfying the following conditions:

$\left(\phi_{1}\right) \phi$ is non-increasing in variables $t_{2}, \ldots, t_{6}$ and non-decreasing in $t_{1}$, 
$\left(\phi_{2}\right)$ there exist $h \in(0,1)$ and $k>1$ with $h k<1$ such that for $u, v \geq 0$, $u \leq k t$ and $\phi(t, v, v, u, u+v, 0) \leq 0$ or $\phi(t, v, u, v, 0, u+v) \leq 0$ imply $t \leq h v$,

$\left(\phi_{3}\right) \phi(t, t, t, 0, t, t)>0$, for all $t>0$.

The following functions are examples of implicit functions $\phi: \mathbb{R}_{+}^{6} \rightarrow \mathbb{R}$ belonging to the set $\Phi$ due to Popa [17] and Imdad and Ali [6].

\section{Example 1.}

$$
\phi\left(t_{1}, t_{2}, t_{3}, t_{4}, t_{5}, t_{6}\right)=t_{1}-h \max \left\{t_{2}, t_{3}, t_{4}, \frac{t_{5}+t_{6}}{2}\right\},
$$

where $h \in(0,1)$.

\section{Example 2.}

$$
\begin{aligned}
& \phi\left(t_{1}, t_{2}, t_{3}, t_{4}, t_{5}, t_{6}\right)=t_{1}-h {\left[a \max \left\{t_{2}, t_{3}, t_{4}, \frac{t_{5}+t_{6}}{2}\right\}\right.} \\
&\left.+(1-a)\left[t_{2}^{2}, t_{3} t_{4}, t_{5} t_{6}, \frac{1}{2} t_{3} t_{6}, \frac{1}{2} t_{4} t_{5}\right]^{\frac{1}{2}}\right],
\end{aligned}
$$

where $h \in(0,1)$ and $0 \leq a \leq 1$.

\section{Example 3.}

$$
\phi\left(t_{1}, t_{2}, t_{3}, t_{4}, t_{5}, t_{6}\right)=t_{1}-h\left[\max \left\{t_{2}^{2}, t_{3} t_{4}, t_{5} t_{6}, t_{4} t_{6}, t_{3} t_{5}\right\}\right]^{\frac{1}{2}},
$$

where $h \in\left(0, \frac{1}{\sqrt{2}}\right)$,

\section{Example 4.}

$$
\phi\left(t_{1}, t_{2}, t_{3}, t_{4}, t_{5}, t_{6}\right)=t_{1}^{3}+t_{2}^{2}+\frac{t_{1}}{1+t_{5} t_{6}}-\left[a t_{2}^{2}+b t_{3}^{2}+c t_{4}^{2}\right],
$$

where $0<a+b+c<1$.

\section{Example 5.}

$$
\phi\left(t_{1}, t_{2}, t_{3}, t_{4}, t_{5}, t_{6}\right)=t_{1}-\alpha\left[\frac{t_{3}^{2}+t_{4}^{2}}{t_{3}+t_{4}}\right]-\beta\left[t_{5}+t_{6}\right]-\gamma t_{2},
$$

where $0<2 \alpha+2 \beta+\gamma<1$.

\section{Example 6.}

$$
\phi\left(t_{1}, t_{2}, t_{3}, t_{4}, t_{5}, t_{6}\right)=t_{1}-\alpha\left[\frac{t_{5}^{2}+t_{6}^{2}}{t_{5}+t_{6}}\right]-\beta\left[t_{3}+t_{4}\right]-\gamma t_{2},
$$

where $0<2 \alpha+2 \beta+\gamma<1$. 


\section{Example 7.}

$$
\phi\left(t_{1}, t_{2}, t_{3}, t_{4}, t_{5}, t_{6}\right)=t_{1}^{p}-a t_{2}^{p}-\left[\frac{c t_{3} t_{4}^{p}+b t_{5} t_{6}^{p}}{t_{3}+t_{4}}\right],
$$

where $1<2 a+c<2,0<2 a+b<2$ and $p \geq 1$.

Certainly, apart from the foregoing examples, there are many more that meet the requirements $\left(\phi_{1}\right),\left(\phi_{2}\right)$ and $\left(\phi_{3}\right)$.

\section{Main Results}

Now, we state and prove our main result.

Theorem 1. Let $(X, d)$ be a complete metric space and $T: X \rightarrow C L(X)$ and $f: X \rightarrow X$ be mappings satisfying

(1) $T(X) \subset f(X)$,

(2) there exists $\phi \in \Phi$ such that

$$
\phi(H(T x, T y), d(f x, f y), d(f x, T x), d(f y, T y), d(f x, T y), d(f y, T x)) \leq 0,
$$

for all $x, y \in X$,

(3) the pair $(T, f)$ is weakly reciprocally continuous and compatible.

Then the mappings $f$ and $T$ have a coincidence point (i.e., $C(T, f) \neq \emptyset$ ). Moreover, the mappings $f$ and $T$ have a common fixed point in $X$ provided that the pair $(T, f)$ is occasionally coincidentally idempotent.

Proof. Let $x_{0}$ be an arbitrary point in $X$. By (1), we choose a point $x_{1}$ in $X$ such that $y_{1}=f x_{1} \in T x_{0}$. By Lemma 1 , one can find a point $y_{2} \in T x_{1}$ such that $d\left(y_{1}, y_{2}\right) \leq k H\left(T x_{0}, T x_{1}\right)$ where $k>1$. Continuing in this way, one can inductively define sequences $\left\{x_{n}\right\},\left\{y_{n}\right\}$ in $X$ as follows:

$$
y_{n+1}=f x_{n+1} \in T x_{n}
$$

such that

$$
d\left(y_{n+1}, y_{n+2}\right) \leq k H\left(T x_{n}, T x_{n+1}\right) .
$$

On using inequality (3.1) with $x=x_{n}$ and $y=x_{n+1}$ and property $\left(\phi_{1}\right)$ of function $\phi \in \Phi$, we have

$$
\begin{gathered}
\phi\left(\begin{array}{c}
H\left(T x_{n}, T x_{n+1}\right), d\left(f x_{n}, f x_{n+1}\right), d\left(f x_{n}, T x_{n}\right), \\
d\left(f x_{n+1}, T x_{n+1}\right), d\left(f x_{n}, T x_{n+1}\right), d\left(f x_{n+1}, T x_{n}\right)
\end{array}\right) \leq 0, \\
\phi\left(\begin{array}{c}
H\left(T x_{n}, T x_{n+1}\right), d\left(y_{n}, y_{n+1}\right), d\left(y_{n}, y_{n+1}\right), \\
d\left(y_{n+1}, y_{n+2}\right), d\left(y_{n}, y_{n+2}\right), 0
\end{array}\right) \leq 0, \\
\phi\left(\begin{array}{c}
H\left(T x_{n}, T x_{n+1}\right), d\left(y_{n}, y_{n+1}\right), d\left(y_{n}, y_{n+1}\right), \\
d\left(y_{n+1}, y_{n+2}\right), d\left(y_{n}, y_{n+1}\right)+d\left(y_{n+1}, y_{n+2}\right), 0
\end{array}\right) \leq 0 .
\end{gathered}
$$


In view of $(3.3),(3.4)$ and $\left(\phi_{2}\right)$, we get

$$
H\left(T x_{n}, T x_{n+1}\right) \leq h d\left(y_{n}, y_{n+1}\right) .
$$

Hence, (3.3) and (3.5) imply

$$
d\left(y_{n+1}, y_{n+2}\right) \leq k h d\left(y_{n}, y_{n+1}\right) .
$$

Since $k h<1$, it follows from (3.6) that $\left\{y_{n}\right\}$ is a Cauchy sequence in $X$ and then by (3.5), that $\left\{T x_{n}\right\}$ is a Cauchy sequence in $C L(X)$. Also, the completeness of the space $X$ implies the completeness of the hyperspace $C L(X)$. Thus, the sequence $\left\{y_{n}\right\}$ converges to some $z \in X$ and $\left\{T x_{n}\right\}$ converges to some $A \in C L(X)$. Also,

$$
\begin{aligned}
d(z, A) & \leq d\left(z, f x_{n}\right)+d\left(f x_{n}, A\right) \\
& \leq d\left(z, f x_{n}\right)+H\left(T x_{n-1}, A\right) \rightarrow 0(\text { as } n \rightarrow \infty) .
\end{aligned}
$$

Since $A$ is closed in $X$, it follows that $z \in A$. Therefore the compatibility of the pair $(f, T)$ implies non-vacuous compatibility, meaning thereby the existence of a sequence $\left\{x_{n}\right\}$ in $X$ such that $\left\{f x_{n}\right\}$ and $\left\{T x_{n}\right\}$ converge to $z \in X$ and $A \in C L(X)$, respectively so that $z \in A$ and $\lim _{n \rightarrow \infty} H\left(T f x_{n}, f T x_{n}\right)=$ 0 . The weak reciprocal continuity of the mappings $f$ and $T$ implies that $\lim _{n \rightarrow \infty} f T x_{n}=f A$ or $\lim _{n \rightarrow \infty} T f x_{n}=T z$.

Case I: Let $\lim _{n \rightarrow \infty} f T x_{n}=f A$. By (3.2), this yields $f f x_{n} \in f T x_{n-1} \rightarrow$ $f A$ as $n \rightarrow \infty$. The compatibility of $f$ and $T$ yields $\lim _{n \rightarrow \infty} H\left(T f x_{n}, f T x_{n}\right)$ $=0$ so that $T f x_{n} \rightarrow f A$ as $n \rightarrow \infty$. On using (3.1) with $x=z$ and $y=f x_{n}$, we get

$$
\begin{aligned}
& \phi\left(H\left(T z, T f x_{n}\right), d\left(f z, f f x_{n}\right), d(f z, T z),\right. \\
& \left.\quad d\left(f f x_{n}, T f x_{n}\right), d\left(f z, T f x_{n}\right), d\left(f f x_{n}, T z\right)\right) \leq 0 .
\end{aligned}
$$

Since $z \in A$, we have $f z \in f A$. Taking the limit as $n \rightarrow \infty$ in (3.8) and using $\left(\phi_{1}\right)$, we have

$$
\phi(d(T z, f z), 0, d(f z, T z), 0,0, d(f z, T z)) \leq 0 .
$$

Since $d(T z, f z) \leq k d(T z, f z)$, and in view of $\left(\phi_{2}\right)$, we get $f z \in T z$. Hence $z$ is a coincidence point of $f$ and $T$, i.e., $C(T, f)$ is non-empty. Also, compatibility of the pair $(T, f)$ implies that $T$ and $f$ commute at their coincidence points, i.e., $f T z=T f z$.

If the mappings $f$ and $T$ are occasionally coincidentally idempotent, then there exists $z^{\prime} \in C(T, f)$ with $f f z^{\prime}=f z^{\prime}$. This implies $f z^{\prime}=f f z^{\prime} \in f T z^{\prime}=$ $T f z^{\prime}$. Hence $f z^{\prime}$ is a common fixed point of the mappings $f$ and $T$. This completes the proof.

Case II: Now assume that $\lim _{n \rightarrow \infty} T f x_{n}=T z$. Then the compatibility of $f$ and $T$ implies $\lim _{n \rightarrow \infty} H\left(T f x_{n}, f T x_{n}\right)=0$, and so $f T x_{n} \rightarrow T z$ as $n \rightarrow \infty$. By (3.2), ff $f x_{n} \in f T x_{n-1} \rightarrow T z$ as $n \rightarrow \infty$ and there exists a point 
$u \in X$ (due to (1)) such that $f u \in T z$. On using (3.1) with $x=f x_{n}$ and $y=u$, we have

$$
\begin{aligned}
& \phi\left(H\left(T f x_{n}, T u\right), d\left(f f x_{n}, f u\right), d\left(f f x_{n}, T f x_{n}\right),\right. \\
& \left.d(f u, T u), d\left(f f x_{n}, T u\right), d\left(f u, T f x_{n}\right)\right) \leq 0 .
\end{aligned}
$$

Passing to the limit as $n \rightarrow \infty$ and using $\left(\phi_{1}\right)$ as well as $f u \in T z$, we get

$$
\phi(d(f u, T u), 0,0, d(f u, T u), d(f u, T u), 0) \leq 0 .
$$

Since $d(T u, f u) \leq k d(T u, f u)$, owing to $\left(\phi_{2}\right)$, we get $f u \in T u$ so that $u$ is the coincidence point of $f$ and $T$. The compatibility of the pair $(T, f)$ implies commutativity of $T$ and $f$ at their coincidence points, i.e., $f T u=T f u$.

If the mappings $T$ and $f$ are occasionally coincidentally idempotent, the rest of the proof runs along the lines of Case I. This completes the proof.

Corollary 1. The conclusions of Theorem 1 remain true if for all distinct $x, y \in X$ the implicit relation (3.1) is replaced by one of the following:

$$
\begin{aligned}
& H(T x, T y) \\
& \leq h \max \left\{d(f x, f y), d(f x, T x), d(f y, T y), \frac{1}{2}[d(f x, T y)+d(f y, T x)]\right\}, \\
& \text { where } h \in(0,1) \text {; } \\
& H(T x, T y) \leq h\left[\left\{d(f x, f y), d(f x, T x), d(f y, T y), \frac{1}{2}[d(f x, T y)+d(f y, T x)]\right\}\right. \\
& +(1-a)\left[d^{2}(f x, f y), d(f x, T x) d(f y, T y), d(f x, T y) d(f y, T x),\right. \\
& \left.\left.\frac{1}{2} d(f x, T x) d(f y, T x), \frac{1}{2} d(f y, T y) d(f x, T y)\right]^{\frac{1}{2}}\right] \\
& \text { where } h \in(0,1) \text { and } 0 \leq a \leq 1 \text {; } \\
& \begin{array}{r}
H(T x, T y) \leq h\left[\operatorname { m a x } \left\{d^{2}(f x, f y), d(f x, T x) d(f y, T y), d(f x, T y) d(f y, T x),\right.\right. \\
d(f y, T y) d(f y, T x), d(f x, T x) d(f x, T y)\}]^{\frac{1}{2}}
\end{array} \\
& \text { where } h \in\left(0, \frac{1}{\sqrt{2}}\right) \text {; } \\
& H^{3}(T x, T y)+d^{2}(f x, f y) \leq a d^{2}(f x, f y)+b d^{2}(f x, T x)+c d^{2}(f y, T y) \\
& -\frac{H(T x, T y)}{1+d(f x, T y) d(f y, T x)},
\end{aligned}
$$


where $0<a+b+c<1$;

$$
\begin{aligned}
& H(T x, T y) \leq \alpha\left[\frac{d^{2}(f x, T x)+d^{2}(f y, T y)}{d(f x, T x)+d(f y, T y)}\right] \\
& +\beta[d(f x, T y)+d(f y, T x)]+\gamma d(f x, f y), \\
& \text { where } 0<2 \alpha+2 \beta+\gamma<1 \text {; } \\
& \begin{aligned}
& H(T x, T y) \leq \alpha\left[\frac{d^{2}(f x, T y)+d^{2}(f y, T x)}{d(f x, T y)+d(f y, T x)}\right] \\
&+\beta[d(f x, T x)+d(f y, T y)]+\gamma d(f x, f y),
\end{aligned} \\
& \text { where } 0<2 \alpha+2 \beta+\gamma<1 \text {; } \\
& H^{p}(T x, T y) \leq\left[\frac{c d(f x, T x) d^{p}(f y, T y)+b d(f x, T y) d^{p}(f y, T x)}{d(f x, T x)+d(f y, T y)}\right]+a d^{p}(f x, f y), \\
& \text { where } 1<2 a+c<2,0<2 a+b<2 \text { and } p \geq 1 \text {. }
\end{aligned}
$$

Proof. The proof in each of the cases easily follows from Theorem 1 in view of Examples 2.1-2.7.

Suppose that $T$ is a self mapping from $X$ to $X$. Then Theorem 1 can be stated for single-valued mappings as follows:

Corollary 2. Let $(X, d)$ be a complete metric space and let $f, T: X \rightarrow X$ satisfy

(1) $T(X) \subset f(X)$,

(2) there exists $\phi \in \Phi$ such that

$$
\phi(d(T x, T y), d(f x, f y), d(f x, T x), d(f y, T y), d(f x, T y), d(f y, T x)) \leq 0,
$$$$
\text { for all } x, y \in X \text {, }
$$

(3) the pair $(f, T)$ is weakly reciprocally continuous and compatible.

If the mappings $f$ and $T$ have a coincidence point (i.e., $C(T, f) \neq \emptyset)$, then the mappings $f$ and $T$ have a unique common fixed point in $X$.

Proof. The proof of this corollary can be completed on the lines of the proof of Theorem 1 contained in Pant et al. [13].

\section{An application}

In this section, we apply Corollary 2 to get a homotopy result. For further details, readers are referred to the book of Agarwal et al. [1]. 
Theorem 2. Let $(X, d)$ be a complete metric space, $F$ be a closed subset of $X, U$ be an open subset of $X$ with $U \subset F$ and $f: F \rightarrow X$ be a mapping. Also suppose that $T: F \times[0,1] \rightarrow X$ is an operator satisfying the following conditions:

(i) $x \neq T(x, t)$ for each $x \in F \backslash U$ and each $t \in[0,1]$,

(ii) there exists $\phi \in \Phi$ such that

$$
\begin{aligned}
\phi(d(T(x, t), T(y, t)), & d(f x, f y), d(f x, T(x, t)), \\
& d(f y, T(y, t)), d(f x, T(y, t)), d(f y, T(x, t))) \leq 0,
\end{aligned}
$$

for all $x, y \in F$ and each $t \in[0,1]$,

(iii) there exist $\alpha, \beta \geq 0$ and a continuous increasing function $\gamma:[0,1] \rightarrow$ $\mathbb{R}$ such that

$$
\begin{gathered}
d\left(T\left(x, t_{1}\right), T\left(x, t_{2}\right)\right) \leq \alpha\left|\gamma\left(t_{1}\right)-\gamma\left(t_{2}\right)\right| \text { and } \\
\max \left\{d\left(T\left(x, t_{1}\right), T\left(y, t_{1}\right)\right), d(f x, f y)\right\} \leq \beta d(x, y),
\end{gathered}
$$

for all $t_{1}, t_{2} \in[0,1]$ and $x, y \in F$,

(iv) $T(X) \subset f(X)$,

(v) the pair $(f, T)$ is weakly reciprocally continuous and compatible,

(vi) $f(\bar{B}(x, R) \subset \bar{B}(x, R)$, for all $x \in F(f)$ and $R>0$.

If $T(\cdot, 0)$ and $f$ have a common fixed point in $F$, then $T(\cdot, 1)$ and $f$ have a common fixed point in $U$.

Proof. Consider the set

$$
Q:=\{(t, x) \in[0,1] \times U: x=f x=T(x, t)\} .
$$

Clearly, $Q$ is a nonempty set. Now, endow $Q$ with the partial order:

$$
(t, x) \preceq(s, y) \quad \text { iff } \quad t \leq s \text { and } d(x, y) \leq \alpha[\gamma(s)-\gamma(t)] .
$$

Next, let $K$ be a totally ordered subset of $Q$ and let $t^{*}=\sup \{t:(t, x) \in$ $K\}$. Consider a sequence $\left\{\left(t_{n}, x_{n}\right)\right\} \subset K$ such that $\left(t_{n}, x_{n}\right) \preceq\left(t_{n+1}, x_{n+1}\right)$ and $t_{n} \rightarrow t^{*}$, as $n \rightarrow \infty$. Then, we get

$$
d\left(x_{m}, x_{n}\right) \leq \alpha\left[\gamma\left(t_{m}\right)-\gamma\left(t_{n}\right)\right],
$$

for all $m, n \in \mathbb{N}$, with $m>n$. Passing to the limit as $m, n \rightarrow \infty$, we obtain $d\left(x_{m}, x_{n}\right) \rightarrow 0$ so that $\left\{x_{n}\right\}$ is a Cauchy sequence and it converges to an element $x^{*} \in X$. Now, $x^{*}=f x^{*}$ as

$$
d\left(x_{n}, f x^{*}\right)=d\left(f x_{n}, f x^{*}\right) \leq \beta d\left(x_{n}, x^{*}\right) .
$$


Also, $x^{*}=T\left(x^{*}, t^{*}\right)$ as

$$
\begin{aligned}
d\left(x_{n}, T\left(x^{*}, t^{*}\right)\right) & =d\left(T\left(x_{n}, t_{n}\right), T\left(x^{*}, t^{*}\right)\right) \\
& \leq d\left(T\left(x_{n}, t_{n}\right), T\left(x_{n}, t^{*}\right)\right)+d\left(T\left(x_{n}, t^{*}\right), T\left(x^{*}, t^{*}\right)\right) \\
& \leq \alpha\left[\gamma\left(t^{*}\right)-\gamma\left(t_{n}\right)\right]+\beta d\left(x_{n}, x^{*}\right) .
\end{aligned}
$$

Now, from (i) we deduce that $x^{*} \in U$, and hence $\left(t^{*}, x^{*}\right) \in Q$. Since $K$ is totally ordered, we get $(t, x) \preceq\left(t^{*}, x^{*}\right)$, for each $(t, x) \in K$. Thus $\left(t^{*}, x^{*}\right)$ is an upper bound of $K$. Consequently, because of Zorn's lemma $Q$ has a maximal element, say $\left(t_{0}, x_{0}\right) \in Q$. To complete the proof, we have to show that $t_{0}=1$.

Suppose on contrary that $t_{0}<1$. Choose $R>0$ and $t \in\left(t_{0}, 1\right]$ such that $B\left(x_{0}, R\right) \subset U$ and $R:=\alpha\left[\gamma(t)-\gamma\left(t_{0}\right)\right]$. Then, we get

$$
\begin{aligned}
d\left(x_{0}, T\left(x_{0}, t\right)\right) & \leq d\left(x_{0}, T\left(x_{0}, t_{0}\right)\right)+d\left(T\left(x_{0}, t_{0}\right), T\left(x_{0}, t\right)\right) \\
& \leq 0+\alpha\left[\gamma(t)-\gamma\left(t_{0}\right)\right] \\
& =R .
\end{aligned}
$$

In view of (vi), it follows that $f, T(\cdot, t): \bar{B}\left(x_{0}, R\right) \rightarrow \bar{B}\left(x_{0}, R\right)$ satisfy all the hypotheses of Corollary 2. Thus, we apply Corollary 2 to deduce the existence of a common fixed point $x \in \bar{B}\left(x_{0}, R\right)$ for $F$ and $T(\cdot, t)$, and hence $(t, x) \in Q$. Finally, as

$$
d\left(x_{0}, x\right) \leq R=\alpha\left[\gamma(t)-\gamma\left(t_{0}\right)\right]
$$

we have $\left(t_{0}, x_{0}\right) \prec(t, x)$, which contradicts the maximality of $\left(t_{0}, x_{0}\right)$. This completes the proof.

Acknowledgements. The third author (Zoran Kadelburg) is grateful to the Ministry of Education, Science and Technological Development of Serbia. The fourth author (Calogero Vetro) is a member of the Gruppo Nazionale per l'Analisi Matematica, la Probabilità e le loro Applicazioni (GNAMPA) of the Istituto Nazionale di Alta Matematica (INdAM).

\section{REFERENCES}

[1] R. P. Agarwal, M. Meehan and D. O'Regan, Fixed Point Theory and Applications, Cambridge Univ. Press, United Kingdom, (2001).

[2] J. P. Aubin and I. Ekeland, Applied Nonlinear Analysis, Wiley, New York, (1984).

[3] V. Berinde and F. Vetro, Common fixed points of mappings satisfying implicit contractive conditions, Fixed Point Theory Appl., (2012), 2012:105.

[4] R. W. Corley, Some hybrid fixed point theorems related to optimization, J. Math. Anal. Appl., 120 (1980), 528-532.

[5] A. Gairola, J. Singh and M. C. Joshi, Coincidence and fixed point for weakly reciprocally continuous single-valued and multi-valued maps, Demonstratio Math., Article in press (2014). 
[6] M. Imdad and J. Ali, A general fixed point theorem for hybrid contractions via implicit functions, South East Asian Bull. Math., 31 (2007), 73-80.

[7] M. Imdad, A. Ahmad and S. Kumar, On nonlinear nonself hybrid contractions, Rad. Mat., 10 (2) (2001), 233-244.

[8] Z. Kadelburg, S. Chauhan and M. Imdad, A hybrid common fixed point theorem under certain recent properties, Sci. World J., 2014 (2014), Article ID 860436, 6 pages.

[9] H. Kaneko, Single-valued and multi-valued $f$-contractions, Boll. Unione Mat. Ital., 4-A, (1985), 29-33.

[10] H. Kaneko, A common fixed point of weakly commuting multi-valued mappings, Math. Jap., 33 (5), (1988), 741-744.

[11] S. B. Nadler Jr., Multivalued contraction mappings, Pacific J. Math., 20 (2) (1969), 457-488.

[12] R. P. Pant, Common fixed points of four mappings, Bull. Cal. Math. Soc., 90 (1998), 281-286.

[13] R. P. Pant, R. K. Bisht and D. Arora, Weak reciprocal continuity and fixed point theorems, Ann. Univ. Ferrara, Sez. VII Sci. Mat., 57 (1), (2011), 181-190.

[14] H. K. Pathak and R. Rodríguez-López, Noncommutativity of mappings in hybrid fixed point results, Bound. Value Probl., (2013), 2013:145, 21 pages.

[15] V. Popa, Fixed point theorems for implicit contractive mappings, Stud. Cerc. St. Ser. Math. Univ. Bacau, 7 (1997), 127-133.

[16] V. Popa, Some fixed point theorems for compatible mappings satisfying an implicit relation, Demonstratio Math., 33 (1999), 157-163.

[17] V. Popa, Coincidence and fixed points theorems for noncontinuous hybrid contractions, Nonlinear Anal. Forum, 7 (2)(2002), 153-158.

[18] V. Popa and A. M. Patriciu, Coincidence and common fixed points for hybrid mappings satisfying an implicit relation and applications, Thai J. Math., submitted.

[19] S. L. Singh, K. S. Ha and Y. J. Cho, Coincidence and fixed points of nonlinear hybrid contractions, Int. J. Math. Math. Sci., 12 (2) (1989), 247-256.

[20] S. L. Singh and S. N. Mishra, Coincidences and fixed points of reciprocally continuous and compatible hybrid maps. Int. J. Math. Math. Sci., 30 (10) (2002), 627-635.

[21] C. Vetro and F. Vetro, Common fixed points of mappings satisfying implicit relations in partial metric spaces, J. Nonlinear Sci. Appl., 6 (3) (2013), 152-161.

[22] R. Wegrzyk, Fixed point theorems for multivalued functions and their applications to functional equations, Diss. Math., (Rozprawy Mat.), 201 (1982), 1-28.

(Received: January 28, 2014)

(Revised: March 28, 2014)

\author{
Sunny Chauhan \\ Near Nehru Training Center \\ H. No. 274, Nai Basti B-14 \\ Bijnor-246701, Uttar Pradesh \\ India \\ sun.gkv@gmail.com \\ Mohammad Imdad \\ Department of Mathematics \\ Aligarh Muslim Univeristy \\ Aligarh 202002 \\ India \\ mhimdad@yahoo.co.in
}


Zoran Kadelburg

Faculty of Mathematics

University of Belgrade, Studentski trg 16

11000 Beograd

Serbia

kadelbur@matf.bg.ac.rs

Calogero Vetro

Dipartimento di Matematica e Informatica Università degli Studi di Palermo

Via Archirafi, 34, 90123 Palermo

Italy

calogero.vetro@unipa.it 